\title{
Decoupling of global brain activity and cerebrospinal fluid flow in
}

\section{Parkinson's cognitive decline}

3 Short running title: fMRI glymphatic and cognitive deficit

4 Feng Han ${ }^{1}$, Gregory L. Brown ${ }^{2,3}$, Yalin Zhu ${ }^{1}$, Aaron E. Belkin-Rosen ${ }^{1}$, Mechelle M. Lewis ${ }^{3,4}$,

5 Guangwei $\mathrm{Du}^{3}$, Yameng Gu${ }^{1}$, Paul J. Eslinger ${ }^{3,5}$, Richard B. Mailman ${ }^{3,4}$, Xuemei Huang ${ }^{3,4,5,6,7,8}$

$6 \quad *, \mathrm{Xiao} \mathrm{Liu}^{1,8} *$.

7 Affiliations:

$8{ }^{1}$ Department of Biomedical Engineering, The Pennsylvania State University, PA, USA;

$9{ }^{2}$ Department of Engineering Science and Mechanics, The Pennsylvania State University, PA, 10 USA;

$11{ }^{3}$ Department of Neurology, Pennsylvania State University Milton S. Hershey Medical Center,

12 Hershey, PA, USA;

$13{ }^{4}$ Department of Pharmacology, Pennsylvania State University Milton S. Hershey Medical Center, 14 Hershey, PA, USA;

$15{ }^{5}$ Department of Radiology, Pennsylvania State University Milton S. Hershey Medical Center,

16 Hershey, PA, USA;

$17{ }^{6}$ Department of Neurosurgery, Pennsylvania State University Milton S. Hershey Medical Center, 18 Hershey, PA, USA;

${ }^{7}$ Department of Kinesiology, Pennsylvania State University Milton S. Hershey Medical Center, Hershey, PA, USA;

$21{ }^{8}$ Institute for Computational and Data Sciences, The Pennsylvania State University, PA, USA;

$23 *$ Correspondence to:

24 Dr. Liu (xx1213@engr.psu.edu) or Dr. Huang (xuemei@psu.edu) 


\section{Abstract}

Background: Deposition and spreading of misfolded proteins ( $\alpha$-synuclein and tau) have

29 been linked to Parkinson's cognitive dysfunction. The glymphatic system may play an important

30 role in the clearance of these toxic proteins via cerebrospinal fluid (CSF) flow through

31 perivascular and interstitial spaces. Recent studies discovered that sleep-dependent global brain

32 activity is coupled to CSF flow that may reflect glymphatic function.

33 Objective: To determine if the decoupling of brain activity-CSF flow is linked to

34 Parkinson's cognitive dysfunction.

Methods: Functional and structural MRI data, clinical motor (Unified Parkinson's Disease

36 Rating Scale), and cognitive (Montreal Cognitive Assessment, MoCA) scores were collected

37 from 60 Parkinson's and 58 control subjects. Parkinson's patients were subgrouped into those

38 with (MoCA < 26; $\mathrm{N}=29$ ) and without (MoCA $\geq 26 ; \mathrm{N}=31$ ) mild cognitive impairment (MCI).

39 The coupling strength between the resting-state global blood-oxygen-level-dependent signal

40 (gBOLD) and associated CSF flow was quantified, compared among groups, and associated with

41 clinical and structural measurements.

42 Results: gBOLD-CSF coupling decreased significantly $(p<0.006)$ in Parkinson's

43 patients showing MCI, compared to those without MCI and controls. Reduced gBOLD-CSF

44 coupling was associated with decreased MoCA scores that was present in Parkinson's patients ( $p$

$45=0.005)$ but not in controls $(p=0.65)$. Weaker gBOLD-CSF coupling in Parkinson's patients

46 also was associated with a thinner right entorhinal cortex (Spearman's correlation $=-0.36 ; p=$

47 0.012), an early structural change often seen in Alzheimer's. 
49 related to Parkinson's cognitive impairment.

51 Keywords: cerebrospinal fluid flow (CSF); cognitive impairment; glymphatic system;

52 global resting-state fMRI signal; Parkinson's disease. 
Parkinson's disease is characterized clinically by motor dysfunction ${ }^{1}$ and pathologically

55 by both $\alpha$-synuclein-positive Lewy bodies/neurites and dopamine neuron loss in the substantia

56 nigra pars compacta of the basal ganglia. ${ }^{2}$ Recent literature also highlights non-motor features of

57 the disease, with cognitive decline being one of the most debilitating symptoms. Indeed, $>75 \%$

58 of Parkinson's patients who survive for more than 10 years ultimately develop dementia. ${ }^{3}$ The

59 exact mechanisms contributing to cognitive decline in Parkinson's disease (PD) are unknown

60 and probably multifactorial. Many pathological processes have been proposed. The aggregation

61 and spreading of $\alpha$-synuclein to extra-nigral subcortical and cortical structures ${ }^{4,5}$ is one

62 prominent hypothesis. Superimposed Alzheimer's disease (AD) pathology ${ }^{6-9}$ could be another

63 cause, since moderate to severe Alzheimer's-co-pathology (e.g., amyloid- $\beta$ and tau) has been

64 found in 51\% of PD patients, and is associated with faster time to dementia than Parkinson's

65 pathology alone. ${ }^{10}$

Increasing evidence suggests an important role of a newly discovered "glymphatic

67 system" in clearing toxic proteins from the brain; ${ }^{11}$ these include $\alpha$-synuclein, ${ }^{12,13}$ amyloid- $\beta,{ }^{14}$

68 and tau. ${ }^{15}$ In this clearance pathway, cerebrospinal fluid (CSF) flows into the interstitial space

69 through the periarterial space, facilitated by astroglial aquaporin- 4 water channels. The bulk CSF

70 flow then drives interstitial fluid (ISF) flux and interstitial solutes/proteins into the perivenous

71 space around deep-draining veins where they eventually are transported into the lymphatic

72 system. ${ }^{11,14,16}$ Intriguingly, the glymphatic system functions mainly during sleep, but much less

73 engaged during the awake state. "This leads to the hypothesis that this "waste clearance"

74 function is one universal mechanism contributing to the need for sleep in all species. This may

75 also explain a diurnal rhythm of amyloid- $\beta$, tau, and $\alpha$-synuclein. ${ }^{15,17,18}$ 
Despite its potential role in cognitive dysfunction and dementias, the study of the

77 glymphatic system in humans has been challenging, largely due to a lack of tools that can assess

78 glymphatic function directly and non-invasively. Two-photon microscopy and fluorescence

79 imaging are used commonly in animal studies of the glymphatic system, but cannot yet be

80 applied to human subjects. ${ }^{19,20}$ Contrast-enhanced MRI has been employed to track whole-brain

81 CSF flow, including in human subjects, ${ }^{21,22}$ but broad application is limited by the need for

82 intrathecal contrast agents and the long-duration experiments. Diffusion and structural MRI have

83 been proposed to probe glymphatic function through surrogate markers such as perivascular

84 space, ${ }^{22-24}$ however, they do not directly reflect dynamic aspects of the glymphatic system.

85 The pioneering study of Kiviniemi et al. ${ }^{25}$ suggested that low-frequency $(<0.1 \mathrm{~Hz})$

86 resting-state fMRI (rsfMRI) blood-oxygen-level-dependent (BOLD) signals are linked to CSF

87 dynamics and, thus, glymphatic function. Additional evidence supports the link between global

88 brain activity (measured by BOLD) and glymphatic function. First, the global BOLD (gBOLD)

89 signal, like glymphatic function, is highly sensitive to brain vigilance state, and also has large-

90 amplitude fluctuations during drowsiness or sleep. ${ }^{26-28}$ Second, the gBOLD signal is

91 accompanied by profound low-frequency $(<0.1 \mathrm{~Hz})$ physiological changes including in cardiac

92 pulsation, ${ }^{29-31}$ respiration, ${ }^{32-34}$ and arterial signals. ${ }^{35}$ These latter physiological functions have

93 been hypothesized to drive glymphatic CSF flow. ${ }^{36-40}$ Third, the large gBOLD signal during

94 sleep was reported to couple to strong CSF movement ${ }^{41}$ that is an essential component of the

95 glymphatic system. Collectively, the resting-state global activity and associated physiological

96 modulations are hypothesized to represent highly coordinated neural and physiological processes

97 closely linked to glymphatic clearance. Thus, the gBOLD-CSF coupling may serve as a marker

98 for gauging glymphatic function. 
There is limited research on the role of the glymphatic system in PD or even in PD

100 animal models, in part because $\alpha$-synuclein conformational changes and accumulation were

101 believed to occur within neurons or at presynaptic terminals. ${ }^{42}$ Recent evidence, however,

102 indicates that $\alpha$-synuclein is secreted into brain extracellular spaces, ${ }^{43}$ suggesting a potential role

103 of glymphatic clearance in PD. Similar to amyloid- $\beta$ and tau, $\alpha$-synuclein in both ISF and CSF

104 increased in response to sleep deprivation. ${ }^{15}$ In addition, tau protein, which can be cleaned by

105 glymphatic system, ${ }^{44}$ plays an important role in the development of PD and PD dementia., ${ }^{7,45,46}$

106 Thus, we hypothesized that coupling between the global brain signal and CSF flow is related to 107 glymphatic function and thus cognitive impairment in Parkinson's patients.

108 Methods

\section{Study design and participants}

110 Sixty PD subjects and 58 controls for whom we had rsfMRI data and Montreal Cognitive

111 Assessment (MoCA) scores were included. The PD subjects were recruited from a tertiary

112 movement disorders clinic and controls from the spouse population of the clinic or via IRB-

113 approved recruitment materials posted in the local community. PD diagnosis was confirmed by a

114 movement disorder specialist according to the UK Brain Bank criteria. ${ }^{47}$ All subjects were free

115 of major/unstable medical issues such as liver, kidney, or thyroid abnormality, and deficiency of

116 vitamin $\mathrm{B}_{12}$, or any cerebrovascular disease or neurological condition (other than PD). Disease

117 duration was obtained from subject history, with onset defined as the first diagnosis by a medical

118 professional. 


\section{Ethics approval}

120 The full protocol of this study was approved by the Institutional Review Board of Human 121 Subjects Protection Office at the Penn State Milton S. Hershey Medical Center College of 122 Medicine. All subjects provided written informed consent. The study was conducted in 123 accordance with the principles of the Declaration of Helsinki.

\section{Clinical assessments}

126 UPDRS-III) were obtained. UPDRS-I evaluates non-motor aspects of daily living, UPDRS-II

127 assesses motor aspects of daily living reported by the subjects, and UPDRS-III evaluates motor

128 symptoms assessed by a trained examiner. ${ }^{48,49}$ Levodopa-equivalent daily dosage (LEDD) was

129 calculated for Parkinson's subjects according to published criteria. ${ }^{50}$ Cognition was assessed

130 using the Montreal Cognitive Assessment (MoCA) ${ }^{51}$ and depression by the Hamilton

131 Depression Rating Scale (HAM-D). ${ }^{52}$ Following procedures reported in previous studies, ${ }^{51,53}$

132 Parkinson's patients were subgrouped into those with (MoCA < 26) and without (MoCA $\geq 26)$

133 mild cognitive impairment (MCI).

\section{Image acquisition and preprocessing}

All MRI data were obtained on a 3.0 Tesla MR scanner (Trio, Siemens Magnetom,

136 Erlangen, Germany, with an 8-channel phased-array head coil). To avoid systematic bias,

137 Parkinson's and control subjects were scanned in an intermixed fashion throughout the study.

138 Each rsfMRI acquisition began with a T1-weighted MPRAGE structural MRI sequence

139 (repetition time/echo time $(\mathrm{TR} / \mathrm{TE})=1540 / 2.3 \mathrm{msec}$, field of view $=256 \mathrm{~mm} \times 256 \mathrm{~mm}$, matrix

$140=256 \times 256$, slice thickness $=1 \mathrm{~mm}$, slice number $=176$ ) that was used for anatomical

141 segmentation and template normalization. In each rsfMRI session, we acquired 240 fMRI 
142 volumes using a 3D echo-planar image (EPI) sequence (flip angle $=90^{\circ}$, spatial resolution $=$

$1433 \times 3 \times 4 \mathrm{~mm}^{3}$, slice thickness $=3.0 \mathrm{~mm}$ ) with $\mathrm{TR} / \mathrm{TE}=2000 / 30 \mathrm{msec}$.

RsfMRI data were preprocessed with a rsfMRI preprocessing pipeline adapted from the

1451000 Functional Connectomes Project (version 1.1-beta). ${ }^{54}$ Specifically, we performed motion

146 correction, skull/edge stripping, spatial smoothing (full width at maximum $(\mathrm{FWHM})=4 \mathrm{~mm})$,

147 bandpass filtering $(0.01-0.1 \mathrm{~Hz})$, and linear and quadratic detrending. In addition, the first five

148 rsfMRI volumes were discarded to avoid unsteady state of magnetization and potential edge

149 effect of temporal filtering. These procedures generated the preprocessed rsfMRI at individual

150 space, from which the gBOLD and CSF signals were extracted. Similar to approach of Fultz et

151 al., ${ }^{41}$ we skipped the nuisance regression of gBOLD and CSF signal since these variables were

152 the focus of the current study. Head motion also may affect the gBOLD signal, ${ }^{34}$ and we thus

153 tested its effects separately by regarding the head motion as a confounding factor as described

154 below. To derive the fMRI-based drowsiness index ${ }^{34}$ (see below), we further registered spatially

155 the rsfMRI images to the standard MNI-152 space ${ }^{55}$ (spatial resolution: $3 \times 3 \times 3 \mathrm{~mm}$ ) and

156 (temporally) standardized signals of each voxel to Z scores.

157 Because there is a great deal of research related to early-stage Alzheimer's disease, ${ }^{56-59}$

158 we quantified entorhinal cortex (ERC) thickness and hippocampal volume from both

159 hemispheres using Free-Surfer (version 5.1.0) from T1-weighted images. ${ }^{60}$ These two markers

160 for early-stage or preclinical Alzheimer's were selected because Parkinson's subjects in our

161 cohort have relatively high MoCA scores (mean MoCA was 24.8) compared to that for

162 diagnosing Alzheimer's (MoCA < 18). ${ }^{61}$ The process included motion correction, tissue

163 classification, brain extraction, registration, and segmentation of gray matter volumetric

164 structures, such as the ERC and hippocampus. To control for inter-subject variability of brain 
165 size, we normalized the ERC thickness and hippocampal volume with estimated total brain tissue

166 volume. $^{62}$

on the Harvard-Oxford cortical and subcortical structural atlases. ${ }^{64}$ To avoid spatial blurring

170 from the registration process, this gray-matter mask was transformed from the MNI-152 space

171 back to the original space of each subject based on the same rationale used in a previous study. ${ }^{41}$

172 The fMRI signal temporally was Z-normalized and then spatially averaged across all gray matter

173 voxels. The resulting gBOLD signal reflects the amplitude and synchronization level of global

174 brain activity. The CSF signal was extracted from the bottom slice of the fMRI acquisition near

175 the bottom of the cerebellum and normalized by the temporal mean to percentage change. This

176 bottom slice is expected to have maximal sensitivity to inflow effects: ${ }^{65} \mathrm{CSF}$ inflow from outside

177 the fMRI acquisition volume, which has not experienced repeated radiofrequency pulses, would

178 increase the fMRI signal. ${ }^{41}$ The mask of CSF regions was obtained manually based on increased

179 image intensity compared to surrounding brain regions in $\mathrm{T} 2 *$-weighted fMRI and confirmed

180 with T1-weighted MRI images.

Following procedures from Fultz et al., ${ }^{41}$ the cross-correlation function first was

182 calculated between the gBOLD and CSF signals to quantify their cross-correlations at different

183 time lags (the gBOLD was regarded as the reference). The gBOLD-CSF cross-correlation at the

184 time lag of +4 seconds, where the negative peak was found, was employed to quantify the

185 gBOLD-CSF coupling for each subject. We also calculated the cross-correlation function

186 between the negative firstorder derivative of the gBOLD signal and the CSF signal to compare

187 with findings from Fultz et al. ${ }^{41}$ We used a permutation method to test the statistical significance 
188 for the above gBOLD-CSF cross-correlations. The gBOLD and CSF signals from different

189 subjects were matched randomly and their cross-correlation functions were computed and

190 averaged. This procedure was repeated 10,000 times to build a null distribution for the mean

191 gBOLD-CSF cross-correlation function, which then was used for statistical inference.

\section{Comparison of $\mathrm{gBOLD}$-CSF coupling and association with clinical metrics}

A linear regression was first conducted between the gBOLD-CSF coupling and age, and

194 the two-sample t test then was then used to compare the gBOLD-CSF coupling between males

195 and females. After these two initial analyses, the age- and gender-adjusted gBOLD-CSF

196 coupling was used for all subsequent analyses described below.

We compared gBOLD-CSF coupling among control, PD-MCI (MoCA < 26), and PD-

non-MCI (MoCA $\geq 26$ ) subjects using the two-sample t-test. We then used Spearman's

199 correlations to quantify associations between gBOLD-CSF coupling and various clinical

200 measures, including MoCA scores, MDS-UPDRS scores, ERC thickness, and hippocampal

201 volumes. These correlational analyses were conducted within the control (if applicable) and PD

202 groups, as well as the entire group of subjects.

We further evaluated the potential effects of multiple factors, including high-leverage

204 data point, disease duration, head motion, and arousal state, on the association between the

205 gBOLD-CSF coupling and MoCA score. We re-ran the correlation between the two after

206 excluding two high-leverage data points with MoCA less than 15, as well as controlling for

207 disease duration. We estimated head motion and drowsiness/sleepiness level of subjects and then

208 re-tested the association between gBOLD-CSF coupling and MoCA controlling for these factors.

209 For head motion, we quantified framewise displacement (FD), is defined as the sum of the 
210 absolute values of six translational and rotational motion parameters. ${ }^{66}$ These values then were

211 averaged within the same session to quantify individual subject head motion. ${ }^{34}$ For the arousal

212 state, we adapted an fMRI-based drowsiness index. ${ }^{34,67}$ Briefly, we computed the spatial

213 correlation between each rsfMRI volume and a pre-defined drowsiness template derived

214 previously ${ }^{28}$ and then took the envelope amplitude of this spatial correlation (i.e., similarity) as

215 an estimation of instantaneous brain arousal level. This drowsiness index then was averaged

216 within each session to represent the overall drowsiness level of each subject and included in the

217 correlation analysis.

$218 \quad$ Statistical analysis

219 In summary, the two-sample t-test was used for group comparisons of continuous

220 demographic and clinical measures, whereas the Fisher exact test was utilized for categorical

221 metrics. $^{68}$ The permutation test was used to assess the statistical significance of the gBOLD-CSF

222 correlations tested. Spearman's correlation was used to quantify inter-subject associations

223 between the gBOLD-CSF coupling and various clinical measures in order to account for

224 potential non-normality of these variables. P-values $<0.05$ were considered statistically

225 significant. We also corrected multiple comparisons using the Bonferroni method. ${ }^{69}$

226 Results

Demographic and clinical data are presented in Table 1. PD participants on average were

ca. 3-years older than controls $(p=0.027)$. The two groups had similar numbers of males and

230 females $(p=0.58)$. PD participants had an average disease duration of $6.6 \pm 5.7$ years, and 
231 significantly higher HAM-D $\left(p=4.3 \times 10^{-5}\right)$ and lower MoCA scores $(p=0.009)$ than controls.

232 There were approximately equal numbers of normal cognition PD patients (MoCA $\geq 26, \mathrm{~N}=31$ )

233 and those with MCI $(\mathrm{MoCA}<26, \mathrm{~N}=29)$.

We first examined whether the gBOLD signal is coupled to strong CSF signal changes in

236 this Parkinson's dataset. As shown in a representative control, changes in the gBOLD signal are

237 associated with large CSF signal changes (Fig. 1B, black and gray arrows). The mean gBOLD-

238 CSF cross-correlation function was characterized by a positive peak $(r=0.28 ; p<0.0001$;

239 permutation test, $N=10,000$ ) around the -4 -sec lag (i.e., correlation with shifting CSF ahead of

240 time by ca. $4 \mathrm{sec})$, whereas there was a negative peak $(r=-0.28 ; p<0.0001)$ at the lag of $+4 \mathrm{sec}$

241 (Fig. 1C, left panel). The cross-correlation function between the CSF signal and the negative

242 first-order derivative of the gBOLD signal displayed a large positive peak at the zero time-lag $(r$

$243=0.37 ; p<0.0001)$. Overall, both cross-correlation functions (Fig. 1C) showed patterns

244 consistent with the data of Fultz et al., ${ }^{41}$ confirming a systematic coupling between the global

245 brain signal and CSF flow.

\section{Comparison of gBOLD-CSF coupling among groups}

We tested whether the gBOLD-CSF coupling was affected by age or gender. A

248 significant correlation was found between age and gBOLD-CSF coupling (Fig. 2A; $\rho=0.32, p<$ 249 0.001), with increased age associated with weaker (less negative) gBOLD-CSF coupling. No 250 significant difference $(p=0.44)$ in gBOLD-CSF coupling was found between males and females. 
251 For scientific rigor, we controlled for the age and gender effects on gBOLD-CSF coupling for all 252 subsequent analyses.

254 showed significantly weaker gBOLD-CSF coupling (Fig. 2B) than controls $(p=0.003)$ and PDnon-MCI $(p=0.006)$ subjects. gBOLD-CSF coupling strength, however, was not different between control and PD-non-MCI subjects $(p=0.96)$.

\section{Association between gBOLD-CSF coupling and cognitive and PD measures} subjects $\left(\rho=-0.36, p=0.005, p_{\text {Bonferroni }}=0.01\right.$, Fig. $\left.2 D\right)$ but not across the controls $(\rho=-0.06, p$

$262=0.65$, Fig. 2C). The Parkinson's-specific gBOLD-CSF coupling/MoCA associations remained

263 significant $\left(\rho=-0.31, p=0.017, p_{\text {Bonferroni }}=0.034\right.$, Fig. $\left.2 \mathbf{E}\right)$ when excluding two subjects with

264 very low MoCA scores. The association is also significant $(p<0.05)$ across the entire group of

265 subjects (Suppl. Fig. 1), or after controlling for disease duration (Suppl. Fig. 2), head motion

266 (Suppl. Fig. 3), and drowsiness score (Suppl. Fig. 4). As seen Suppl. Fig. 5, there was no 267 significant correlation found between the gBOLD-CSF coupling and any of the MDS-UPDRS 268 motor (UPDRS-II, $p=0.80$, UPDRS-III, $p=0.27$ ); or non-motor subscores UPDRS-I, $p=0.49$ ).

Weaker (less negative) gBOLD-CSF coupling was associated significantly with a thinner

271 right ERC region $\left(\rho=-0.36, p=0.012, p_{\text {Bonferroni }}=0.024\right.$, Fig. 3A), whereas only a trend was

272 found for the left ERC thickness $\left(\rho=-0.19, p=0.19, p_{\text {Bonferroni }}=0.38, \quad\right.$ Fig. 3B $)$. Weaker 
273 gBOLD-CSF coupling was not associated with lower hippocampal volume bilaterally $(p>0.05$;

274 Suppl. Figs. 6A-B). There was no correlation between gBOLD-CSF coupling and ERC

275 thickness or hippocampal volume in the control group ( $p>0.05$; see Suppl. Fig. 7).

276

277

278

279

280

281

282

283

284

285

286

287

288

289

290

291

292

293

294

\section{Discussion}

The current study demonstrated a strong coupling between global brain activity and CSF flow using rsfMRI data obtained both from Parkinson's and control subjects, consistent with the idea that gBOLD-CSF coupling is related to glymphatic function. The strength of this gBOLD-

CSF coupling was significantly lower in those Parkinson's patients with cognitive impairment compared to either controls or Parkinson's patients without cognitive dysfunction. Moreover, the gBOLD-CSF coupling strength was correlated with cognitive scores, but only in Parkinson's patients and not controls, suggesting it is linked to Parkinson's-related processes. No correlation was found between gBOLD-CSF coupling and UPDRS motor or non-motor scores, suggesting it is related specifically to a cognitive component of Parkinson's. The reduced gBOLD-CSF coupling also was associated with a thinner ERC that has been observed in early-stage Alzheimer's, suggesting a possible contribution from Alzheimer's-like pathology in PD-MCI. These findings suggest that gBOLD-CSF coupling is a potential noninvasive marker for the glymphatic function that may be related to dementia pathology.

\section{gBOLD-CSF coupling may be related to the sleep-dependent glymphatic system}

Resting-state gBOLD-CSF coupling likely results from highly coordinated neural and physiological processes that are linked closely to glymphatic clearance. This potential relationship is supported by at least three lines of evidence. First, the resting-state gBOLD signal shows a similar sleep dependency as glymphatic function, and is strongly promoted by 
295 drowsiness and sleep. ${ }^{26,27,70-72}$ Sleep deprivation ${ }^{71}$ and hypnotic drugs (e.g., midazolam), ${ }^{73}$

296 significantly enhance the gBOLD signal, whereas caffeine has the opposite effect. ${ }^{72}$ It became $^{2}$

297 clear recently that the strong gBOLD signal fluctuation reflects transient arousal modulations of

298 10-20 sec. $^{28}$ Second, this gBOLD signal is accompanied by slow $(<0.1 \mathrm{~Hz})$, but strong

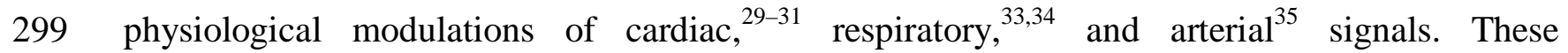

300 physiological modulations have been hypothesized to be the main drivers of glymphatic CSF

301 flow. ${ }^{36-40}$ It is likely that they are mediated through the autonomic system via transient arousal

302 variations underlying the gBOLD signal. ${ }^{29}$ In at least one mouse model (for cerebral amyloid

303 angiopathy), the low-frequency modulations in vessel tone has been linked to amyloid- $\beta$

304 clearance. $^{74}$ Thus, the gBOLD signal and associated physiological modulations should provide

305 sleep-dependent dynamics for CSF flow that are essential for the glymphatic system. Indeed, we

306 found that strong CSF movements coupled to the large gBOLD signal, ${ }^{41}$ adding a new piece of

307 evidence for their relevance to the glymphatic system. This coupling relationship was confirmed

308 and extended in the present study of Parkinson's patients. Overall, this potential link to the

309 glymphatic system could be the primary explanation for the association we found between

310 gBOLD-CSF coupling and cognitive dysfunction in Parkinson's.

313 clearance of toxic misfolded proteins such as $\alpha$-synuclein, amyloid- $\beta$ and tau, thereby possibly

314 contribute to the cognitive decline in PD patients. The initial test of this hypothesis in the current

315 study indicated that gBOLD-CSF coupling was decreased significantly only in Parkinson's

316 patients with cognitive decline. It is also correlated with cognitive MoCA scores, but not with

317 motor and other non-motor symptoms measured by MDS-UPDRS scores. Together, the data 
318 provide the first evidence that gBOLD-CSF coupling may serve as a new, non-invasive,

319 functional metric for quantifying cognitive decline, and warrants future studies of its relationship

320 to the ultimate development of dementia in PD.

\section{gBOLD-CSF coupling may reflect aspects of Alzheimer's pathology}

The present study showed that weaker gBOLD-CSF coupling was associated with a

323 thinner right ERC, although correlations with left ERC thickness or bilateral hippocampal

324 volumes did not reach statistical significance. Right ERC thinning and early loss of ERC

325 symmetry has been suggested a marker for preclinical Alzheimer's. ${ }^{75,76}$ A more pronounced loss

326 in right ERC thickness also is seen with aging. ${ }^{77}$ In Alzheimer's patients, gray matter loss in the

327 right ERC has been shown to occur earlier and be larger than in other brain regions. ${ }^{75}$ In fact, the

328 ERC is known to be the earliest affected brain region in both Alzheimer's and Parkinson's. ${ }^{78-80}$

329 Thus, decreased gBOLD-CSF coupling may be an indicator of early cognitive decrements, and

330 its correlation with cognitive impairment in PD-MCI may be attributed partly to structural

331 changes similar to those in in early Alzheimer's.

\section{Limitations and future directions}

The present study linked the gBOLD-CSF coupling to cognitive dysfunction in PD, but

334 one clear limitation of the present study was that it did not directly link the gBOLD-CSF

335 coupling to accumulation of toxic chemical species (proteins or small molecules). This provides

336 a fertile area for mechanistic understanding of the association of gBOLD-CSF coupling with

337 cognitive impairment. Future studies should also explore other imaging markers that result from

338 the same low-frequency global brain activity and physiological modulations, and relate them to

339 cognitive decline and dementias. Related experiments could include quantification of $\alpha$ - 
340 synuclein and tau (either with PET imaging or CSF measurements), metabolomic studies of CSF,

341 and longitudinal studies of how gBOLD-CSF coupling relates to the prognosis and progression

342 of Parkinson's disease dementia. Finally, despite the recent evidence, the relationship between

343 gBOLD-CSF coupling and the glymphatic system remains hypothetical, and direct evidence

344 using both murine and non-human primate models will be very important.

\section{Acknowledgements}

346 We thank Jiaxuan Diao for preparing some figures and reviewing the manuscript.

\section{Author Roles}

348 Research project: A. Conception, B. Organization, C. Execution;

349 Statistical Analysis: A. Design, B. Execution, C. Review and Critique;

350 Manuscript Preparation: A. Writing of the first draft, B. Review and Critique;

$351 \quad$ FH: $1 \mathrm{~A}, 1 \mathrm{~B}, 1 \mathrm{C}, 2 \mathrm{~B}, 2 \mathrm{C}, 3 \mathrm{~A}$;

$352 \quad$ GLB: $1 \mathrm{C}, 2 \mathrm{C}, 3 \mathrm{~A}, 3 \mathrm{~B}$;

$353 \quad$ YZ: 1C, 2B;

$354 \quad$ AEBR: 1C, 2B;

$355 \quad$ MML: 2C, 3A, 3B;

356 GD: 2C, 3B;

$357 \quad$ YG: 1 C, 2C;

358 PJR: 3B; 
RBM: 2C, 3B;

XH: 1A, 1B, 2A, 2C, 3B;

XL: 1A, 1B, 2A, 2C, 3A, 3B.

\section{Financial disclosure and conflict of interest statement}

363 This work was supported by the National Institutes of Health (NIH) Pathway to

364 Independence Award (K99/R00 5R00NS092996-03), the Brain Initiative award

365 (1RF1MH123247-01), and the NIH R01 award (1R01NS113889-01A1). This work was also

366 supported by the National Institute of Neurological Disorders and Stroke Parkinson's Disease

367 Biomarker Program (NS06722 and NS112008 to X.H), the National Institute of Aging

368 (AG067651 to G.B), the Hershey Medical Center General Clinical Research Center (National

369 Center for Research Resources, Grant UL1 RR033184 that is now at the National Center for

370 Advancing Translational Sciences, Grant UL1 TR000127), the PA Department of Health

371 Tobacco CURE Funds, and the Penn State Translational Brain Research Center. The authors

372 report no financial interests or potential conflicts of interest. 


\section{References}

375 1. Parkinson J. An essay on shaking palsy. 1817; 14: 223- 236.

$376 \quad 2 . \quad$ Venda LL, Cragg SJ, Buchman VL, Wade-Martins R. $\alpha$-Synuclein and dopamine at the crossroads

377 of Parkinson's disease. Trends Neurosci. 2010; 33: 559- 568.

378 3. Aarsland D, Kurz MW. The epidemiology of dementia associated with Parkinson disease. $J$.

$379 \quad$ Neurol. Sci. 2010; 289: 18- 22.

380 4. Mattila PM, Rinne JO, Helenius H, Dickson DW, Röyttä M. Alpha-synuclein-immunoreactive

381 cortical Lewy bodies are associated with cognitive impairment in Parkinson's disease. Acta

382 Neuropathol. 2000; 100: 285- 290.

383 5. Hurtig HI, Trojanowski JQ, Galvin J, et al. Alpha-synuclein cortical Lewy bodies correlate with

384 dementia in Parkinson's disease. Neurology. 2000; 54: 1916- 1921.

385 6. Lei P, Ayton S, Finkelstein DI, et al. Tau protein: Relevance to Parkinson's disease. Int. J.

386 Biochem. Cell Biol. 2010; 42: 1775- 1778.

387 7. Zhang X, Gao F, Wang D, et al. Tau pathology in Parkinson's disease. Front. Neurol. 2018 ; 9.

388 8. Goris A, Williams-Gray CH, Clark GR, et al. Tau and $\alpha$-synuclein in susceptibility to, and

389 dementia in, Parkinson's disease. Ann. Neurol. 2007; 62: 145- 153.

390 9. Vermersch P, Delacourte A, Javoy $\square$ Agid F, Hauw J $\square$ J, Agid Y. Dementia in Parkinson’s disease:

391 Biochemical evidence for cortical involvement using the immunodetection of abnormal tau

392 proteins. Ann. Neurol. 1993; 33: 445- 450.

393 10. Irwin DJ, Grossman M, Weintraub D, et al. Neuropathological and genetic correlates of survival 394 and dementia onset in synucleinopathies: a retrospective analysis. Lancet Neurol. 2017; 16: 55-65.

395 11. Jessen NA, Munk ASF, Lundgaard I, Nedergaard M. The Glymphatic System: A Beginner's 396 Guide. Neurochem. Res. 2015; 40: 2583-2599. 
397 12. Sundaram S, Hughes RL, Peterson E, et al. Establishing a framework for neuropathological

398 correlates and glymphatic system functioning in Parkinson's disease. Neurosci. Biobehav. Rev.

399 2019; 103: 305- 315.

400 13. Valdinocci D, Radford RAW, Goulding M, et al. Extracellular interactions of Alpha-synuclein in multiple system atrophy. Int. J. Mol. Sci. 2018; 19: 4219.

14. Tarasoff-Conway JM, Carare RO, Osorio RS, et al. Clearance systems in the brain - Implications for Alzheimer disease. Nat. Rev. Neurol. 2015; 11: 457- 470.

404 15. Holth JK, Fritschi SK, Wang C, et al. The sleep-wake cycle regulates brain interstitial fluid tau in 405 mice and CSF tau in humans. Science (80-. ). 2019; 363: 880- 884.

406 16. Mestre H, Mori Y, Nedergaard M. The Brain's Glymphatic System: Current Controversies. Trends $407 \quad$ Neurosci. 2020; 43: 458- 466.

408 17. Musiek ES, Xiong DD, Holtzman DM. Sleep, circadian rhythms, and the pathogenesis of $409 \quad$ Alzheimer disease. Exp. Mol. Med. 2015; 47: e148.

410 18. Xie L, Kang H, Xu Q, et al. Sleep drives metabolite clearance from the adult brain. Science (80-. ). $411 \quad 2013 ; \mathbf{3 4 2 :} 373-377$.

412 19. Iliff JJ, Wang M, Liao Y, et al. A paravascular pathway facilitates CSF flow through the brain 413 parenchyma and the clearance of interstitial solutes, including amyloid $\beta$. Sci. Transl. Med. 2012; $414 \quad 4: 147$.

415 20. Iliff JJ, Lee H, Yu M, et al. Brain-wide pathway for waste clearance captured by contrast416 enhanced MRI. J. Clin. Invest. 2013; 123: 1299- 1309.

417 21. Eide PK, Ringstad G. MRI with intrathecal MRI gadolinium contrast medium administration: a 418 possible method to assess glymphatic function in human brain. Acta Radiol. Open. 2015; 4: 1-5.

419 22. Ringstad G, Valnes LM, Dale AM, et al. Brain-wide glymphatic enhancement and clearance in 
humans assessed with MRI. JCI insight. 2018; 3: e121537.

421 23. Taoka T, Masutani Y, Kawai H, et al. Evaluation of glymphatic system activity with the diffusion

422 MR technique: diffusion tensor image analysis along the perivascular space (DTI-ALPS) in

$423 \quad$ Alzheimer's disease cases. Jpn. J. Radiol. 2017; 35: 172- 178.

424 24. Harrison IF, Siow B, Akilo AB, et al. Non-1 invasive imaging of CSF-mediated brain clearance

425 pathways via assessment of perivascular fluid movement with diffusion tensor MRI. Elife. 2018; 7:

426 e34028.

427 25. Kiviniemi V, Wang X, Korhonen V, et al. Ultra-fast magnetic resonance encephalography of 428 physiological brain activity-Glymphatic pulsation mechanisms? J. Cereb. Blood Flow Metab. $429 \quad 2016 ; 36: 1033-1045$.

430 26. Fukunaga M, Horovitz SG, van Gelderen P, et al. Large-amplitude, spatially correlated 431 fluctuations in BOLD fMRI signals during extended rest and early sleep stages. Magn. Reson. 432 Imaging. 2006; 24: 979- 972.

433 27. Horovitz SG, Fukunaga M, De Zwart JA, et al. Low frequency BOLD fluctuations during resting $434 \quad$ wakefulness and light sleep: A simultaneous EEG-fMRI study. Hum. Brain Mapp. 2008; 29: 671435682.

436 28. Liu X, De Zwart JA, Schölvinck ML, et al. Subcortical evidence for a contribution of arousal to $437 \quad$ fMRI studies of brain activity. Nat. Commun. 2018; 9: 395.

438 29. Özbay PS, Chang C, Picchioni D, et al. Contribution of systemic vascular effects to fMRI activity 439 in white matter. Neuroimage. 2018; 176: 541- 549.

440 30. Shmueli K, van Gelderen P, de Zwart JA, et al. Low-frequency fluctuations in the cardiac rate as a 441 source of variance in the resting-state fMRI BOLD signal. Neuroimage. 2007; 38: 306- 320.

442 31. Chang C, Cunningham JP, Glover GH. Influence of heart rate on the BOLD signal: The cardiac 
response function. Neuroimage. 2009; 44: 857- 869.

444 32. Birn RM, Diamond JB, Smith MA, Bandettini PA. Separating respiratory-variation-related fluctuations from neuronal-activity-related fluctuations in fMRI. Neuroimage. 2006; 31: 15361548.

447 33. Power JD, Plitt M, Gotts SJ, et al. Ridding fMRI data of motion-related influences: Removal of signals with distinct spatial and physical bases in multiecho data. Proc. Natl. Acad. Sci. U. S. A. 2018; 115: E2105- E2114.

450 34. Gu Y, Han F, Sainburg LE, Liu X. Transient Arousal Modulations Contribute to Resting-State 451 Functional Connectivity Changes Associated with Head Motion Parameters. Cereb. Cortex. 2020; 30: $5242-5256$.

453 35. Tong Y, Yao J (Fiona), Chen JJ, Frederick B de B. The resting-state fMRI arterial signal predicts 454 differential blood transit time through the brain. J. Cereb. Blood Flow Metab. 2019; 39: 1148$455 \quad 1160$.

456 36. Schley D, Carare-Nnadi R, Please CP, Perry VH, Weller RO. Mechanisms to explain the reverse 457 perivascular transport of solutes out of the brain. J. Theor. Biol. 2006; 238: 962- 974.

458 37. Iliff JJ, Wang M, Zeppenfeld DM, et al. Cerebral arterial pulsation drives paravascular CSF459 Interstitial fluid exchange in the murine brain. J. Neurosci. 2013; 33: 18190- 18199.

460 38. Klose U, Strik C, Kiefer C, Grodd W. Detection of a relation between respiration and CSF pulsation with an echoplanar technique. J. Magn. Reson. Imaging. 2000; 11: 438- 444.

462 39. Yamada S, Miyazaki M, Yamashita Y, et al. Influence of respiration on cerebrospinal fluid movement using magnetic resonance spin labeling. Fluids Barriers CNS. 2013; 10: 36.

464 40. Mestre H, Tithof J, Du T, et al. Flow of cerebrospinal fluid is driven by arterial pulsations and is $465 \quad$ reduced in hypertension. Nat. Commun. 2018; 9: 4878. 
466 41. Fultz NE, Bonmassar G, Setsompop K, et al. Coupled electrophysiological, hemodynamic, and cerebrospinal fluid oscillations in human sleep. Science (80-. ). 2019; 366: 628- 631.

42. Stefanis L. $\alpha$-Synuclein in Parkinson's disease. Cold Spring Harb. Perspect. Med. 2012; 2: a009399.

470 43. Emmanouilidou E, Elenis D, Papasilekas T, et al. Assessment of $\alpha$-synuclein secretion in mouse and human brain parenchyma. PLoS One. 2011; 6: e22225.

472 44. Harrison IF, Ismail O, Machhada A, et al. Impaired glymphatic function and clearance of tau in an Alzheimer's disease model. Brain. 2020; 143: 2576-2 593.

474 45. Wills J, Jones J, Haggerty T, et al. Elevated tauopathy and alpha-synuclein pathology in postmortem Parkinson's disease brains with and without dementia. Exp. Neurol. 2010; 225: 210-

477 46. Irwin DJ, Lee VMY, Trojanowski JQ. Parkinson's disease dementia: Convergence of $\alpha$-synuclein, tau and amyloid- $\beta$ pathologies. Nat. Rev. Neurosci. 2013; 14: 626- 636.

479 47. Hughes AJ, Daniel SE, Kilford L, Lees AJ. Accuracy of clinical diagnosis of idiopathic Parkinson's disease: A clinico-pathological study of 100 cases. J. Neurol. Neurosurg. Psychiatry.

48. Goetz CG, Fahn S, Martinez-Martin P, et al. Movement disorder society-sponsored revision of the unified Parkinson's disease rating scale (MDS-UPDRS): Process, format, and clinimetric testing plan. Mov. Disord. 2007; 22: 41-47.

49. Lewis MM, Harkins E, Lee EY, et al. Clinical Progression of Parkinson's Disease: Insights from the NINDS Common Data Elements. J. Parkinsons. Dis. 2020; 10: 1075- 1085. in Parkinson's disease. Mov. Disord. 2010; 25: 2649- 2653. 
51. Nasreddine ZS, Phillips NA, Bédirian V, et al. The Montreal Cognitive Assessment, MoCA: A brief screening tool for mild cognitive impairment. J. Am. Geriatr. Soc. 2005; 53: 695- 699.

52. Williams JBW. A Structured Interview Guide for the Hamilton Depression Rating Scale. Arch. Gen. Psychiatry. 1988; 45: 742- 747.

53. Dalrymple-Alford JC, MacAskill MR, Nakas CT, et al. The MoCA: Well-suited screen for cognitive impairment in Parkinson disease. Neurology. 2010; 75: 1717- 1725.

54. Biswal BB, Mennes M, Zuo XN, et al. Toward discovery science of human brain function. Proc. Natl. Acad. Sci. U. S. A. 2010; 107: 4734- 4739.

55. Mazziotta JC, Toga AW, Evans A, Fox P, Lancaster J. A probabilistic atlas of the human brain: Theory and rationale for its development. Neuroimage. 1995; 2: 89- 101.

499 56. Babiloni C, Frisoni GB, Pievani M, et al. Hippocampal volume and cortical sources of EEG alpha rhythms in mild cognitive impairment and Alzheimer disease. Neuroimage. 2009; 44: 123- 135.

501 57. Velayudhan L, Proitsi P, Westman E, et al. Entorhinal cortex thickness predicts cognitive decline in Alzheimer's disease. J. Alzheimer's Dis. 2013; 33: 755- 766.

503 58. Fjell AM, Westlye LT, Grydeland H, et al. Accelerating cortical thinning: Unique to dementia or universal in aging? Cereb. Cortex. 2014; 24: 919- 934.

505 59. Ma X, Li Z, Jing B, et al. Identify the atrophy of Alzheimer's disease, mild cognitive impairment and normal aging using morphometric MRI analysis. Front. Aging Neurosci. 2016; 8: 243.

507 60. Fischl B. FreeSurfer. Neuroimage. 2012; 62: 774- 781.

508 61. Hoops S, Nazem S, Siderowf AD, et al. Validity of the MoCA and MMSE in the detection of MCI and dementia in Parkinson disease. Neurology. 2009; 75: 478.

510 62. Smith SM, Zhang Y, Jenkinson M, et al. Accurate, robust, and automated longitudinal and cross511 sectional brain change analysis. Neuroimage. 2002; 17: 479- 489. 
512 63. Aquino KM, Fulcher BD, Parkes L, Sabaroedin K, Fornito A. Identifying and removing widespread signal deflections from fMRI data: Rethinking the global signal regression problem. Neuroimage. 2020; 212: 116614.

64. Desikan RS, Ségonne F, Fischl B, et al. An automated labeling system for subdividing the human cerebral cortex on MRI scans into gyral based regions of interest. Neuroimage. 2006; 31: 968- 980.

65. Gao JH, Liu HL. Inflow effects on functional MRI. Neuroimage. 2012; 62: 1035- 1039.

66. Power JD, Barnes KA, Snyder AZ, Schlaggar BL, Petersen SE. Spurious but systematic correlations in functional connectivity MRI networks arise from subject motion. Neuroimage.

67. Chang C, Leopold DA, Schölvinck ML, et al. Tracking brain arousal fluctuations with fMRI. Proc. Natl. Acad. Sci. U. S. A. 2016; 113: 4518- 4523.

68. Fisher RA. On the Interpretation of $\chi 2$ from Contingency Tables, and the Calculation of P. J. R. Stat. Soc. 1922; 85: 87- 94. 2012; 59: 2142- 2154 . Super. di Sci. Econ. e Commer. di Firenze. 19366; 1493- 1494.

527 70. Liu TT, Nalci A, Falahpour M. The global signal in fMRI: Nuisance or Information? Neuroimage. 2017; 150: 213- 229.

529 71. Yeo BTT, Tandi J, Chee MWL. Functional connectivity during rested wakefulness predicts vulnerability to sleep deprivation. Neuroimage. 2015; 111: 147- 158.

531 72. Wong CW, Olafsson V, Tal O, Liu TT. The amplitude of the resting-state fMRI global signal is related to EEG vigilance measures. Neuroimage. 2013; 83: 983-990.

533 73. Kiviniemi VJ, Haanpää H, Kantola JH, et al. Midazolam sedation increases fluctuation and synchrony of the resting brain BOLD signal. Magn. Reson. Imaging. 2005; 23: 531- 537. 
535 74. van Veluw SJ, Hou SS, Calvo-Rodriguez M, et al. Vasomotion as a Driving Force for

536 Paravascular Clearance in the Awake Mouse Brain. Neuron. 2020; 105: 549- 561.

537 75. Thompson PM, Hayashi KM, De Zubicaray G, et al. Dynamics of gray matter loss in Alzheimer's 538 disease. J. Neurosci. 2003; 23: 994-1005.

539 76. Long X, Zhang L, Liao W, Jiang C, Qiu B. Distinct laterality alterations distinguish mild cognitive 540 impairment and Alzheimer's disease from healthy aging: Statistical parametric mapping with high 541 resolution MRI. Hum. Brain Mapp. 2013; 34: 3400- 3410.

542 77. Hasan KM, Mwangi B, Cao B, et al. Entorhinal Cortex Thickness across the Human Lifespan. $J$. $543 \quad$ Neuroimaging. 2016; 26: 278- 282.

544 78. Braak H, Braak E. The human entorhinal cortex: normal morphology and lamina-specific 545 pathology in various diseases. Neurosci. Res. 1992; 15: 6-31.

546 79. Braak H, Braak E. Neuropathological stageing of Alzheimer-related changes. Acta Neuropathol. $547 \quad$ 1991; 82: 239- 259 .

548 80. Braak H, Del Tredici K, Rüb U, et al. Staging of brain pathology related to sporadic Parkinson's 549 disease. Neurobiol. Aging. 2003; 24: 197- 211. 


\section{Figures}

A

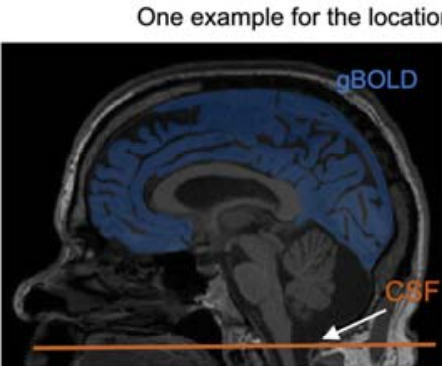

B

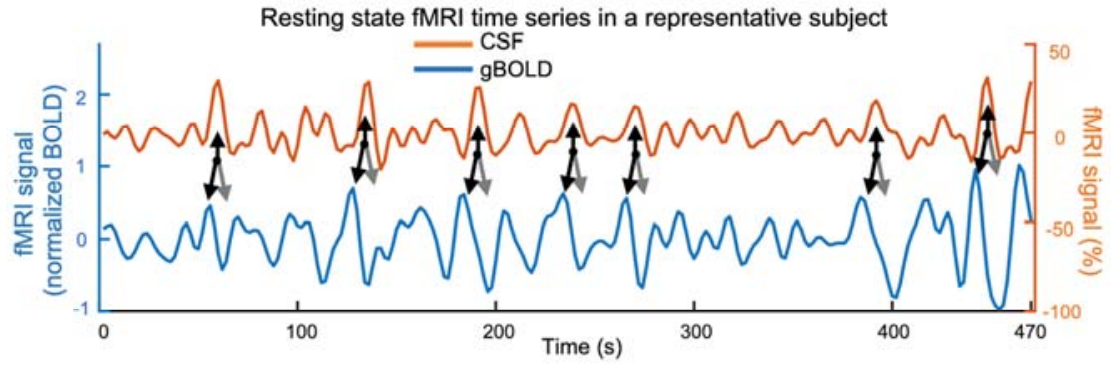

gBOLD vs. CSF FMRI

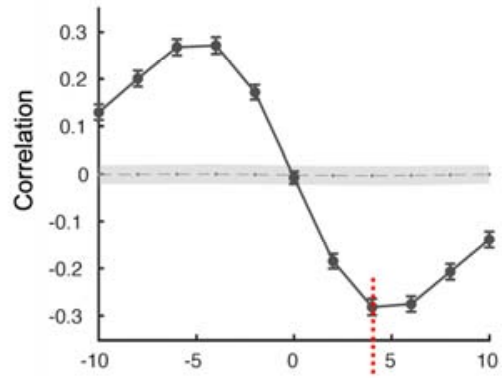

$\operatorname{Lag}(\mathbf{s})$
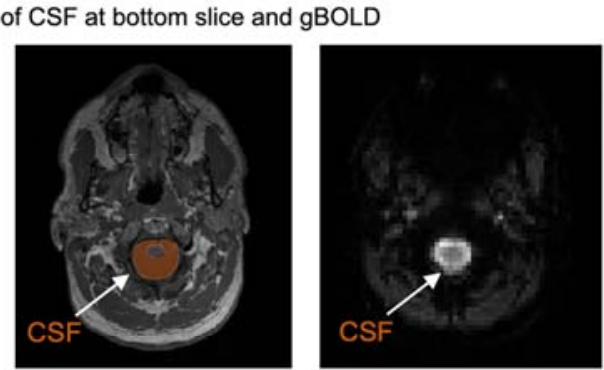

-d/dt gBOLD vs. CSF fMRI

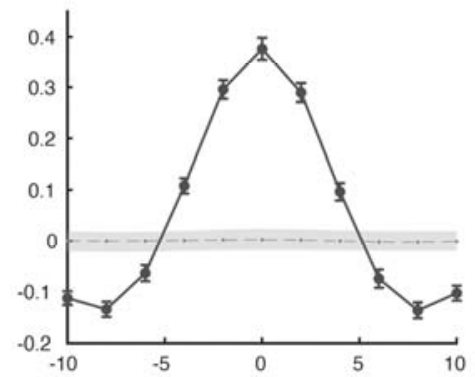

Fig. 1. Systematic coupling between the gBOLD and CSF signals measured by rsfMRI. (A)

554 The gBOLD and CSF fMRI signals were extracted from the whole-brain gray matter regions (the blue mask on a T1-weighted structural MRI in the left panel) and the CSF region at the bottom

556 slice of the fMRI acquisition (the bright region in the right panel and the orange-shaded area in

557 the middle panel), respectively. (B) The gBOLD and CSF signal from a representative subject

558 (control) showed corresponding changes of large amplitude. Large CSF peaks (upwards black

559 arrows) often are preceded by a large positive gBOLD peak (downwards black arrows) and

560 followed by a large negative gBOLD peak (gray arrows). (C) The averaged cross-correlation

561 function ( $\mathrm{N}=118$ subjects) between the gBOLD signal (reference) and the CSF signal (left), as

562 well as between the first-order negative derivative of gBOLD signal (reference) and the CSF 563 signal (right). The gray dashed line and the shaded region mark 95\% confidence intervals for the 564 mean correlation computed on shuffled signals (see Materials and methods for details). The 
565 cross-correlations between the gBOLD and CSF signal at the +4 seconds lag (mean $r:-0.28 ; p<$

566 0.0001, permutation test with $\mathrm{N}=10,000$; red dashed line) was used to represent "the gBOLD-

567 CSF coupling" for subsequent analyses.

568

569 
A

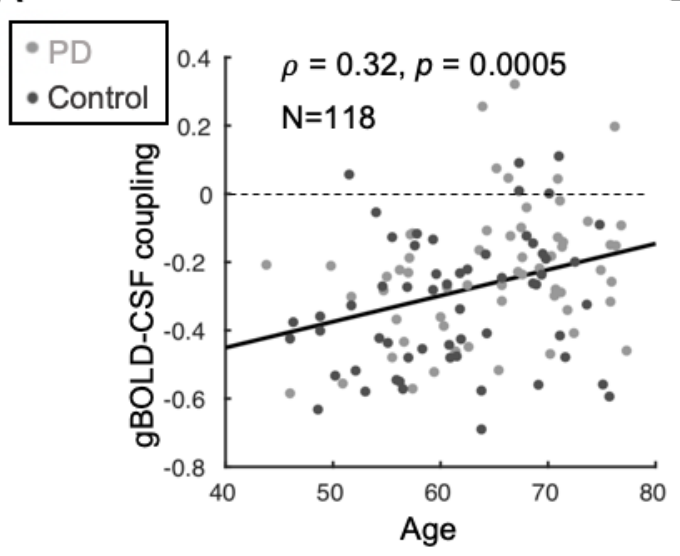

B

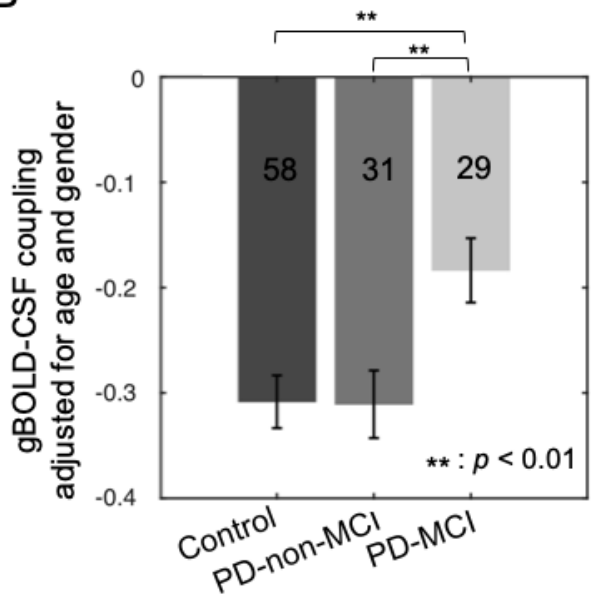

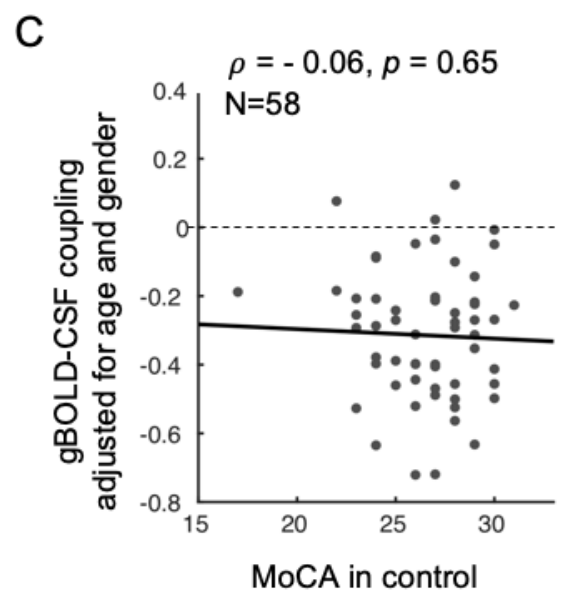

C

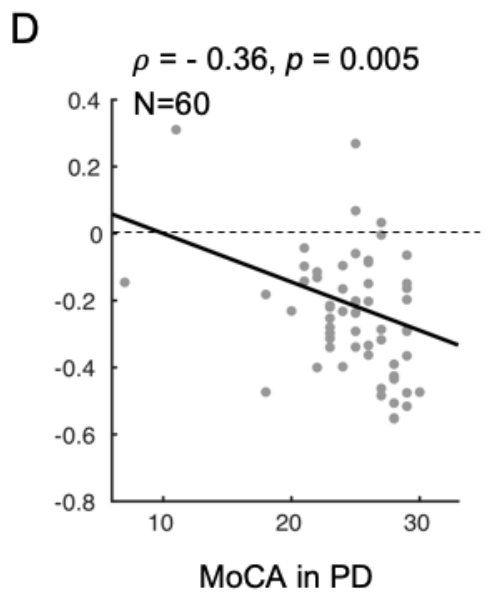

E

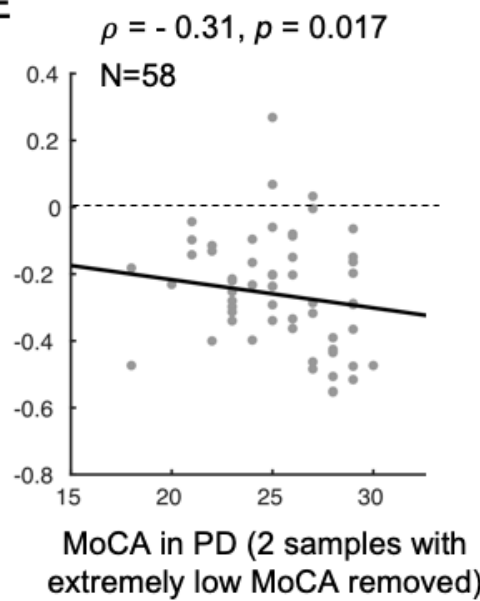

Fig. 2. The associations of gBOLD-CSF coupling to age, disease condition, and MoCA. (A) Older subjects have a weaker (less negative) gBOLD-CSF coupling (Spearman's $\rho=0.32, p=$ 0.0005). (B) Compared with controls and PD-non-MCI subjects, PD-MCI subjects have decreased gBOLD-CSF coupling strength (adjusted for age and gender; $p \leq 0.006$, two-sample ttest). (C-D) The correlation between the gBOLD-CSF coupling (adjusted for age and gender) and MoCA scores is significant in the PD group $\left(\rho=-0.36, p=0.005, p_{\text {Bonferroni }}=0.01\right.$; D) but not in the controls $(\rho=-0.06, p=0.65 ; \mathbf{C})$. (E) The significant correlation between gBOLD-CSF coupling and MoCA within PD subjects remained significant when removing the two subjects with extremely low MoCA scores $\left(\rho=-0.31, p=0.017, p_{\text {Bonferroni }}=0.034\right)$. The dashed lines indicate where the gBOLD-CSF coupling equals zero. 
A

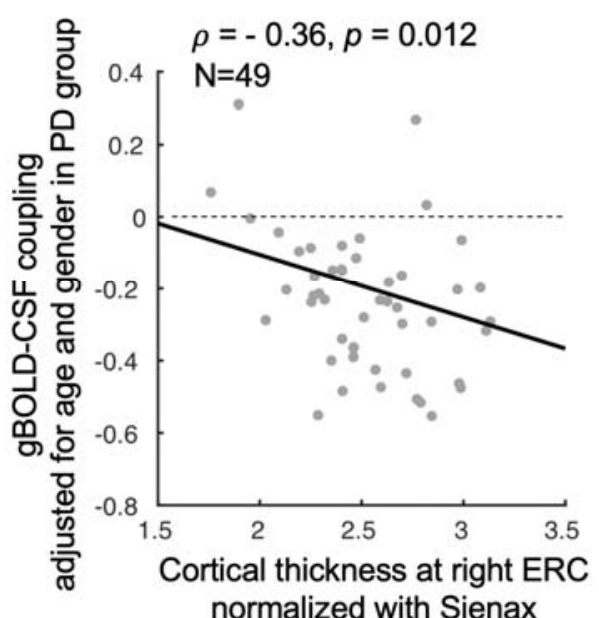

B

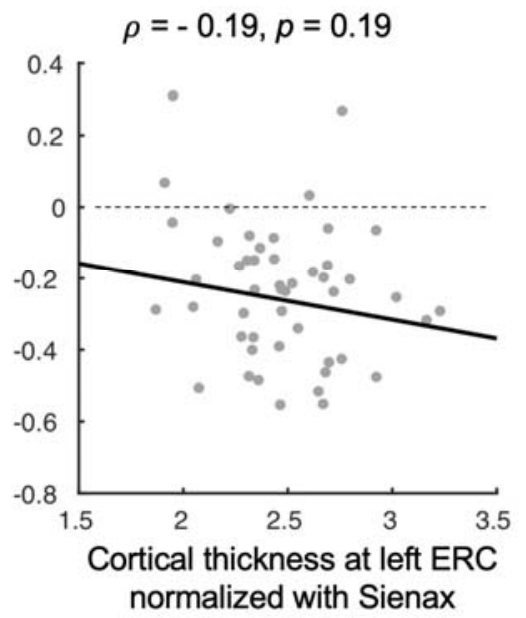

583 Fig. 3. Associations between gBOLD-CSF coupling and thickness of the entorhinal cortex

584 in PD patients. The PD patients with thinner right ERC tend to have weaker gBOLD-CSF

585 coupling (Spearman's $\left.\rho=-0.36, p=0.012, p_{\text {Bonferroni }}=0.024\right)(\mathbf{A})$. This association is similar but

586 not significant $\left(\rho=-0.19, p=0.19, p_{\text {Bonferroni }}=0.38\right)$ for the left ERC $(\mathbf{B})$. The ERC thickness

587 was normalized with the Sienax index. ${ }^{62}$ 
Table 1: Characteristics of the cohort

\begin{tabular}{|l|c|c|c|}
\hline & Controls (N=58) & PD (N=60) & P value \\
\hline Age, y & $61.4(8.0)$ & $64.7(8.2)$ & 0.027 \\
\hline Gender (Male/ Female) & $31 / 27$ & $6 / 24$ & 0.58 \\
\hline Disease duration, y & - & $6.6(5.7)$ & - \\
\hline UPDRS-I & - & $10.6(7.9)$ & - \\
\hline UPDRS-II & - & $9.4(8.5)$ & - \\
\hline UPDRS-III & - & $22.2(20.0)$ & - \\
\hline LEDD, mg & - & $736(487)$ & 0.009 \\
\hline MoCA & $26.5(2.6)$ & $24.8(4.1)$ & $4.3 \times 10^{-5}$ \\
\hline HAM-D & $4.0(3.3)$ & $7.8(4.6)$ & - \\
\hline
\end{tabular}

589 Data represent the mean and standard deviation (in parentheses) unless otherwise indicated.

590 Pairwise comparisons were performed based on the two-sample t-test for all measures except for

591 gender that used the Fisher exact test.

592 Abbreviation: PD, Parkinson's disease group; UPDRS: Unified Parkinson's Disease Rating

593 Scale; LEDD: levodopa equivalent daily dose; MoCA: Montreal Cognitive Assessment; HAM-D:

594 Hamilton Depression Rating Scale. 


\section{Supplemental files}

A

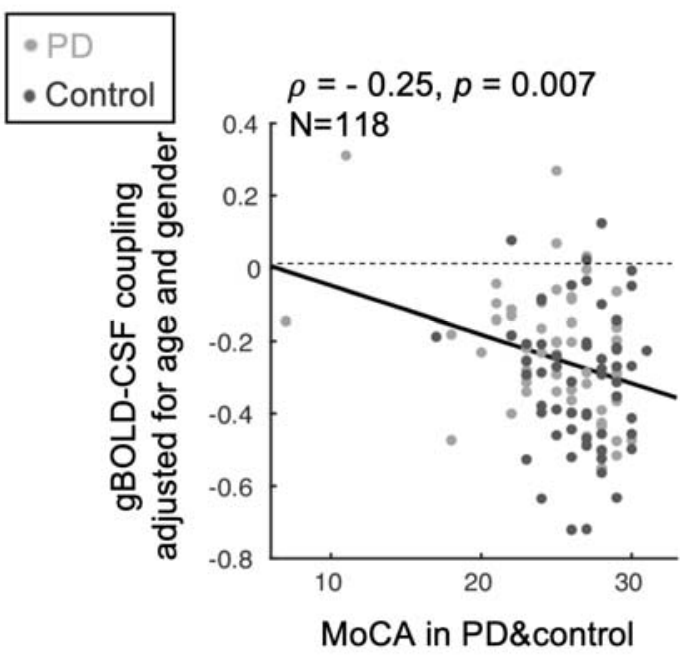

\section{B}

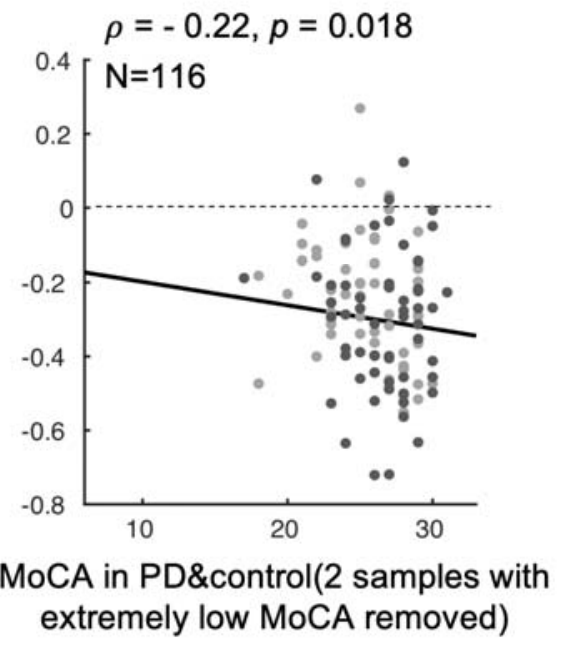

598 Suppl. Fig. 1. The association between the gBOLD-CSF coupling and MoCA remains

599 significant $(p<0.018)$ in the entire cohort of subjects $(\mathbf{A})$ and after removing two high leverage

600 samples (from two PD subjects) with extremely low MoCA scores (B).

601 
A

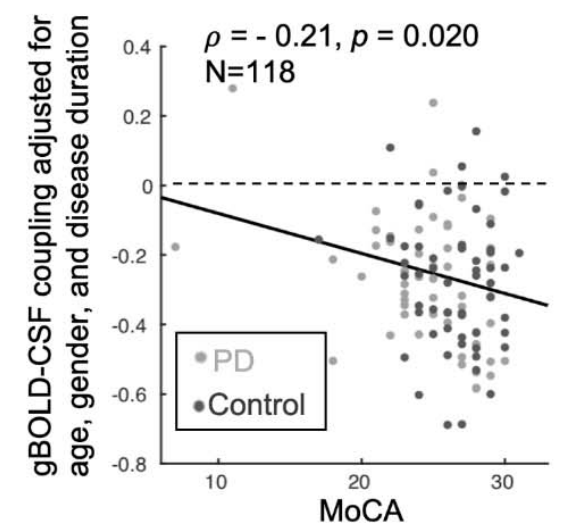

B

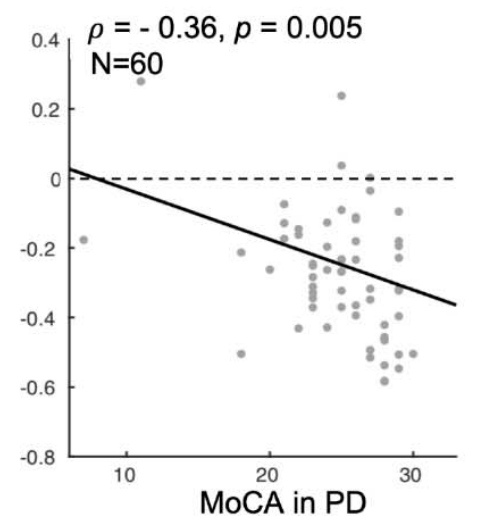

C

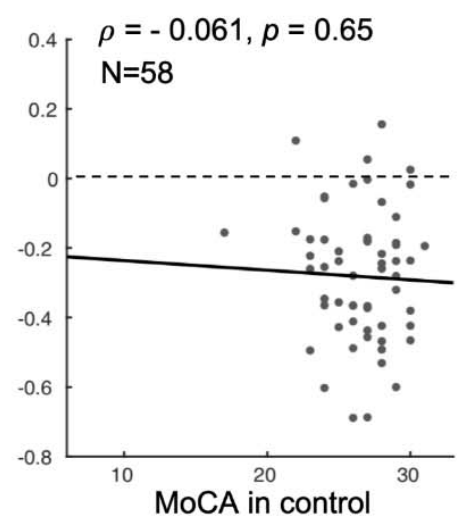

604 Suppl. Fig. 2. The associations between the gBOLD-CSF coupling and MoCA remains

605 significant $(p<0.05)$ for both the entire group of subjects $(\mathbf{A})$ and the PD group (B), after

606 controlling the age, gender, and disease duration, while this significant correlation cannot be

607 found in controls (C).

608 

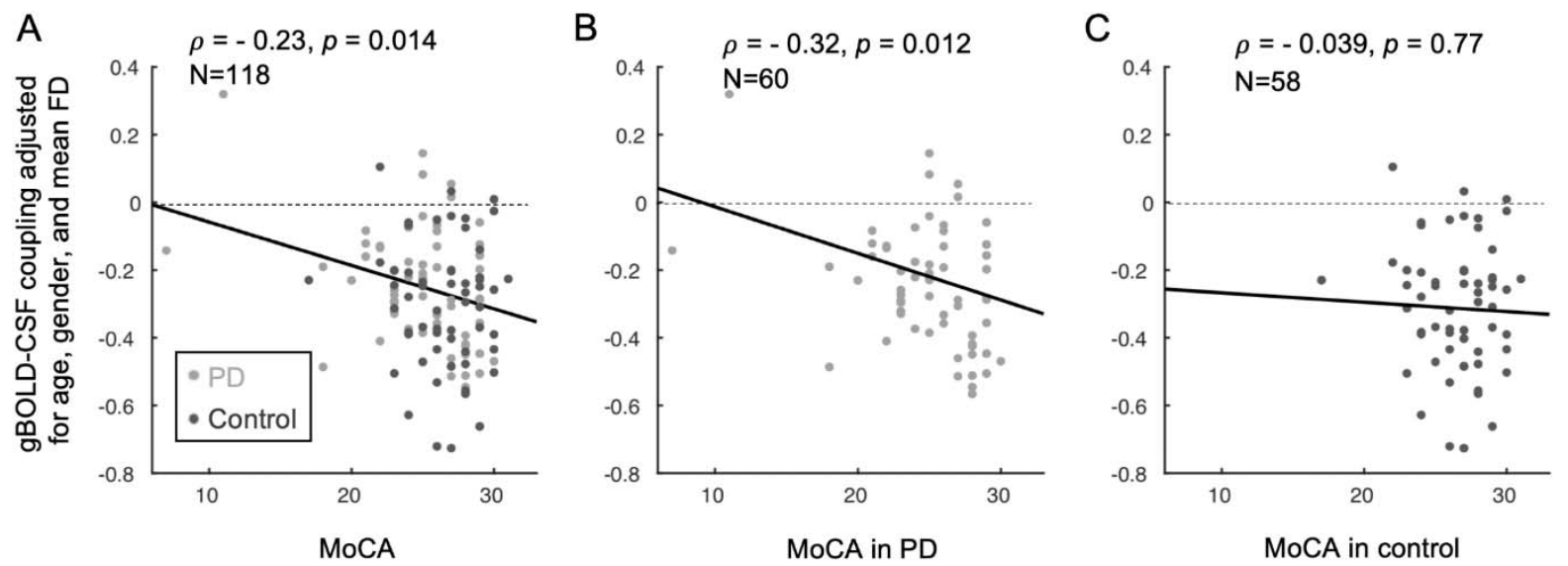

610

611 Suppl. Fig. 3. The association between the gBOLD-CSF coupling and MoCA remains

612 significant $(p<0.05$, corrected) for both the overall group of subjects $(\mathbf{A})$ and within Parkinson's

613 subjects (B), after controlling the age, gender, and head motion, whereas the correlation was

614 absent in controls (C).

615

616 
A

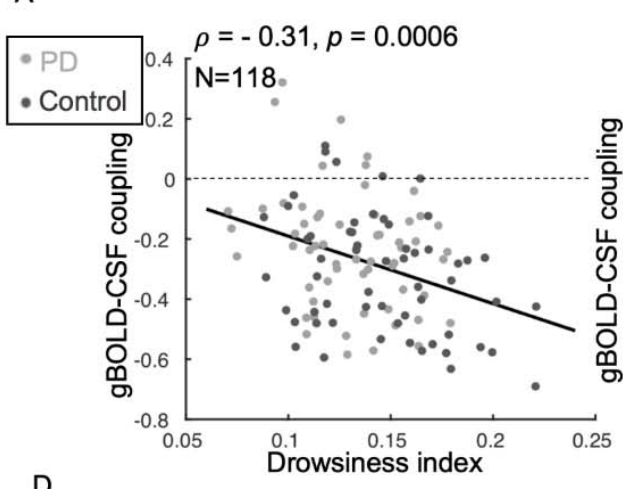

D

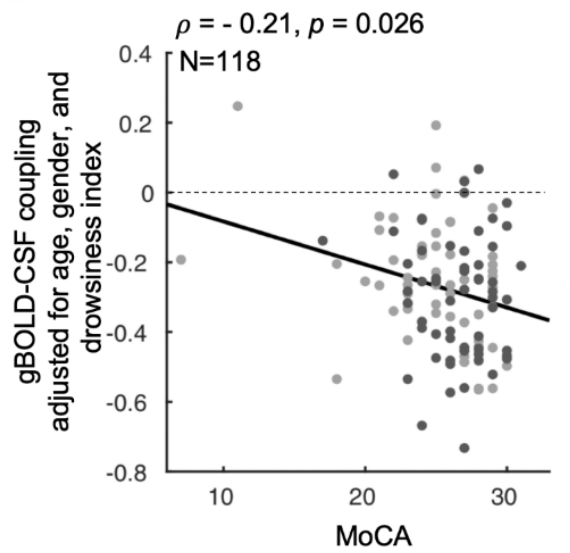

B
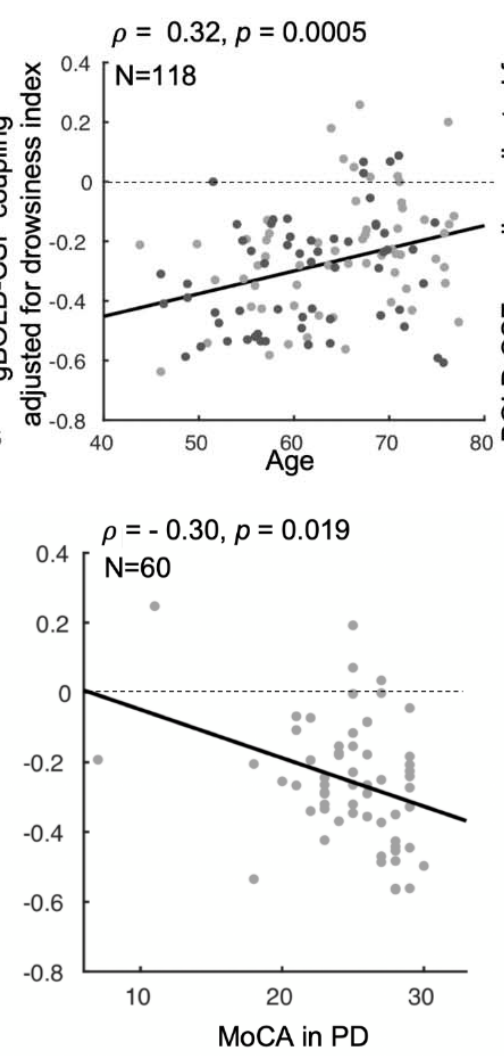

C
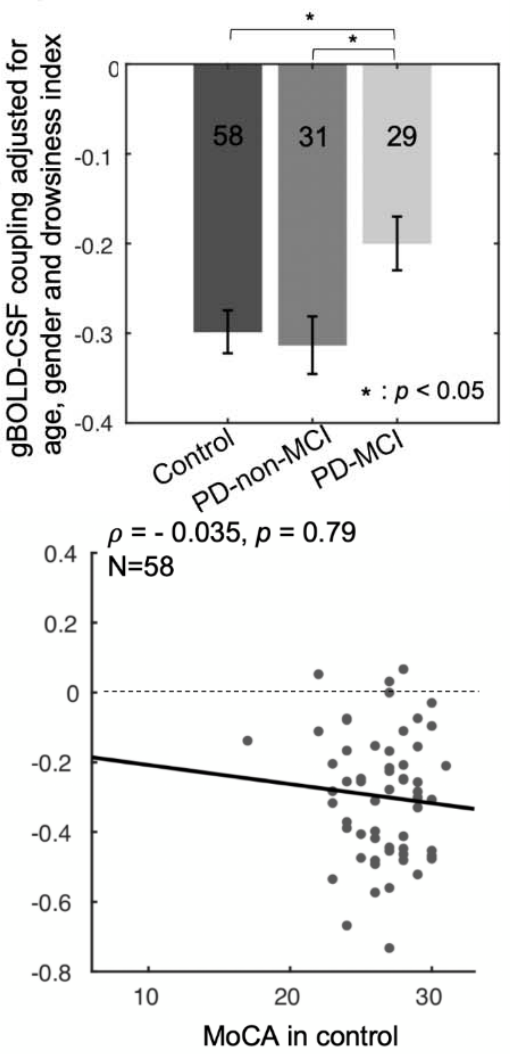

618 Suppl. Fig. 4. The association between the sleep-dependent gBOLD-CSF coupling and age,

619 group, and MoCA scores is not driven by changes in the drowsiness index. (A) The gBOLD-

620 CSF coupling was correlated significantly with the drowsiness index (increased drowsiness

621 index corresponds to a drowsier state) in all subjects, which is quantified based on the similarity

622 between the rsfMRI time-series and a drowsiness template. (B) When adjusted for the

623 drowsiness index, weaker gBOLD-CSF coupling strength remained significantly associated with

624 increasing age $(\rho=0.32, p<0.001)$. (C) PD-MCI subjects had decreased gBOLD-CSF coupling

625 compared to control or PD-non-MCI after adjusting for the drowsiness index (both $p \leq 0.014$ ).

626 (D) Similar to Fig. 2C, gBOLD-CSF coupling (age, gender, and drowsiness index adjusted)

627 remained significantly correlated with MoCA scores across both the entire group of subjects and 
bioRxiv preprint doi: https://doi.org/10.1101/2021.01.08.425953; this version posted January 9,2021 . The copyright holder for this preprint

(which was not certified by peer review) is the author/funder, who has granted bioRxiv a license to display the preprint in perpetuity. It is made available under aCC-BY-NC-ND 4.0 International license.

628 within Parkinson's but not for controls. The dashed lines indicate where the gBOLD-CSF

629 coupling equals zero. 
A

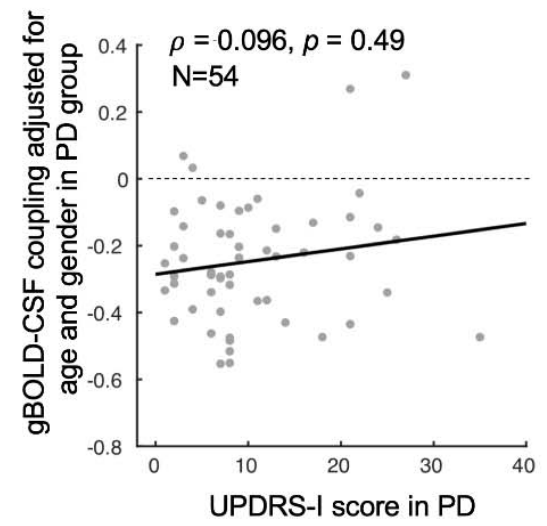

B

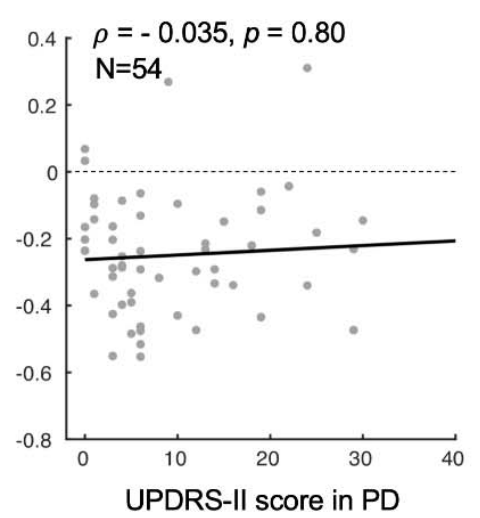

C

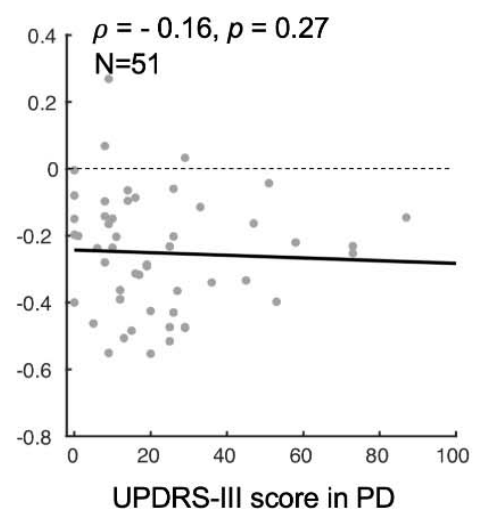

631 Suppl. Fig. 5. The associations (linear regression) between the gBOLD-CSF coupling (adjusted

632 for age and gender) and MDS-UPDRS scores were not significant $(p>0.05)$ within Parkinson's

633 subjects. The linear regression lines were estimated based on least-squares fitting.

634 
A

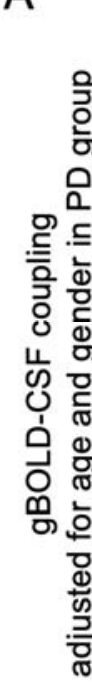

$$
\rho=-0.15, p=0.31
$$

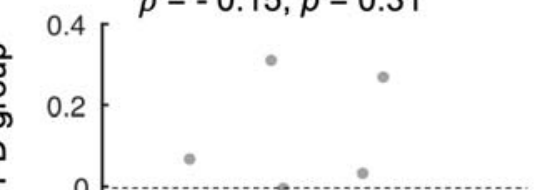

$-0.2$

$-0.4$

$-0.6$

$-0.8$

兽

B

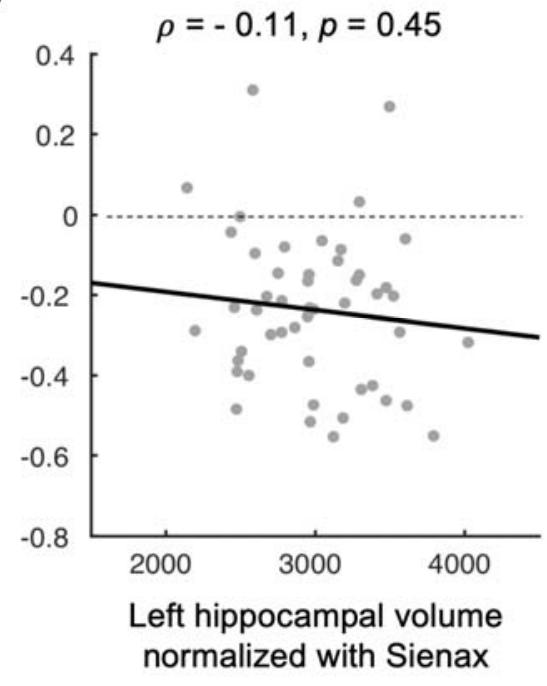

636 Suppl. Fig. S6. Associations between gBOLD-CSF coupling and structural measures of the

637 hippocampus in PD patients. (A-B) The gBOLD-CSF coupling showed a negative correlation

638 with the bilateral hippocampal volumes (normalized with the Sienax index), ${ }^{62}$ but none of the 639 correlations were statistically significant $(p>0.05)$. 
A

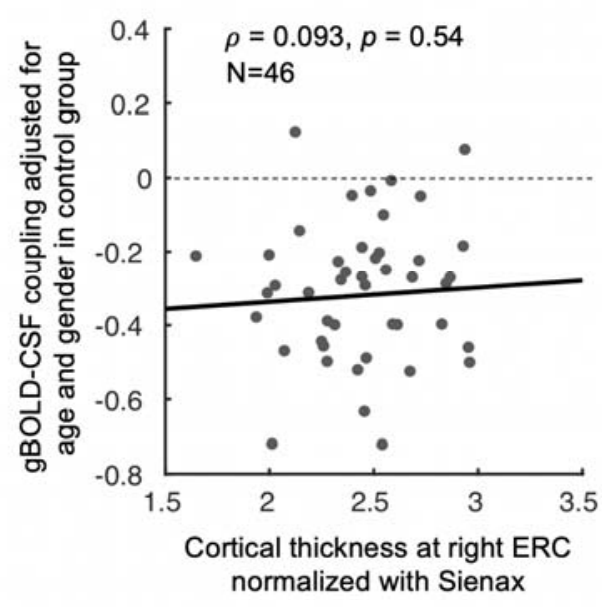

C

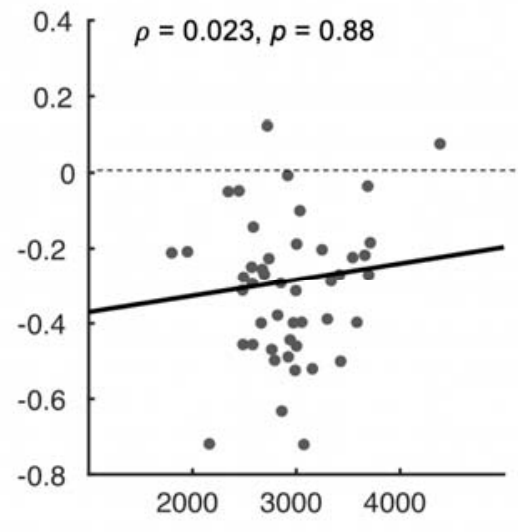

Right hippocampal volume normalized with Sienax
B

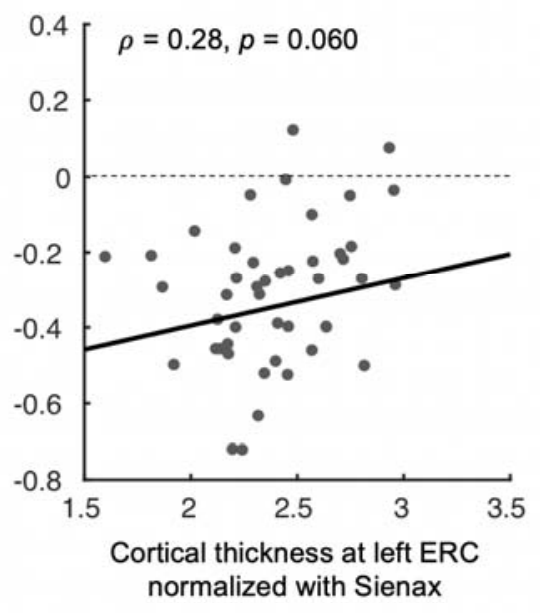

D

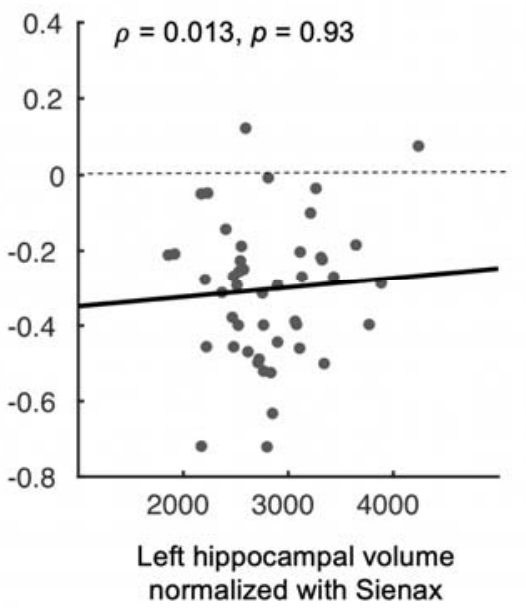

643 Suppl. Fig. S7. gBOLD-CSF coupling and structural measures in the entorhinal cortex and

644 hippocampus were not correlated in controls (all $p>0.05$ ). The linear regression lines were

645 estimated based on least-squares fitting. 